\title{
ROOT CANAL MORPHOLOGY; CONCEPT IN MANDIBULAR 3RD MOLAR BY CONVENTIONALLY ENDODONTIC TREATMENT
}

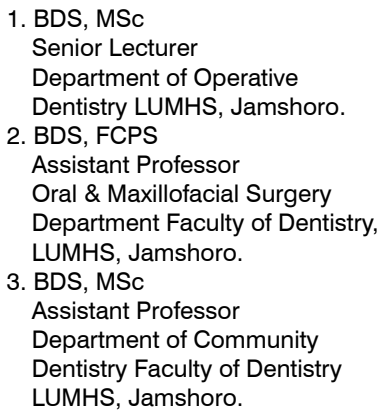

Corresponding Author Dr Naveen Khawaja Dental Section, Aga khan Maternal \& Child Care Centre Qasimabad, Hyderabad. naveen_500@hotmail.com

Article received on: 24/08/2016

Accepted for publication: 28/02/2017

Received after proof reading: 06/04/2017

\section{Dr. Naveen Khawaja ${ }^{1}$, Dr. Suneel Kumar Punjabi ${ }^{2}$, Dr. Munir Ahmed Banglani ${ }^{3}$}

\begin{abstract}
Morphological features of mandibular 3rd molar are always unpredictable, and show a discrepancy by way of different individual. Nevertheless, restorative, prosthetic and orthodontic concerns of these mandibular third molars require root canal treatment in turn to preserve functional elements in the jaw. The variation in the root canal anatomy presents clinical challenges and difficulties for clinician to undertake endodontic therapy. Therefore it's very essential for practitioners must have adequate knowledge of the internal morphology of root canal system, use all techniques, equipments is mandatory to treat the entire root canal system. Study Design: Cross-sectional. Setting: Department: Operative Dentistry, Faculty of Dentistry, Liaquat University of Medical \& Health Sciences, Jamshoro. Period: February 2014 to March 2016. Methodology: Overall 200 patients of mandibular 3rd molar (fully erupted in the jaw) were enrolled with indicative irreversible pulpitis, Were endodontically treated by conventional method using stainless steel hand files, contra-angle small head hand peace by postgraduate trainee. After opening of access cavity, every canal was positioned radiographically by the hand files placed within the canals. Results: Among 200 patients of mandibular third molars were evaluated by conventionally endodontic treatment, Out of 200 patients were found to have a practical errors in the 65 cases and rest of the 135 cases were treated without any errors. Number of canal configuration has found in lower 3rd molar teeth, one canal contained in $4(2.0 \%)$ teeth, two canals in $33(16.5 \%)$ teeth, three canals in $160(80 \%)$ teeth and four in $3(1.5 \%)$ teeth. Conclusion: Mandibular third molars showed huge anatomic irregularity. Number of canals has provided by this study to the practitioner with an understanding of the clinical recommended for lowers third molars.
\end{abstract}

Key words: $\quad$ Mandibular third molar, Root canal, Morphology, Conventional

Article Citation: Khawaja N, Punjabi SK, Banglani MA. Root canal morphology; concept in mandibular 3rd molar by conventionally endodontic treatment. Professional Med J 2017;24(4):617-621. DOI: 10.17957/TPMJ/17.3599

\section{INTRODUCTION}

The prime objective of root canal treatment is to prevent or alleviate the apical periodontitis from mechanical \& chemical cleaning, shaping, and disinfection of the entire pulp space that allow obturation in 3-dimensional of the root canal system \& coronal seal. ${ }^{1,2,3}$

Anatomically divided root canal system into two parts: the pulp chamber, located in crown of the tooth, \& root canal situated in the root of the tooth. ${ }^{4}$ It's a great important to understands the different variations in root canal system which help in detecting the root canals and their management throughout the root canal treatment. $5,6,7,8$ In the molar series mandibular third molar is very last tooth in the jaw, associated with various discrepancy in esteem to number of roots and canals. ${ }^{9,10}$

The variations in the root canal anatomy presents clinical challenges and difficulties for clinician to undertake endodontic therapy. ${ }^{11}$ Once this multifarious anatomy has been identified, the result is directly associated with the eradication \& prevention of microbial contagion. ${ }^{12}$ Most important causative factor of the root canal failure is missing canals or presence of an untreated canal, as dentist fail to search it. Therefore it's very essential for practitioners must have adequate knowledge of the internal morphology of root canal system, use all techniques, equipments is mandatory to treat the entire root canal system. ${ }^{10,13}$ 
From Diagnostic point of view two or more radiographs at various angles, analysis of the pulp chamber floor by a sharp explorer, troughing of grooves by ultrasonic tips, magnification, staining, accomplishing the sodium hypochlorite 'champagne bubble' test, envisioning canal bleeding points, and Cone Beam Computed Tomography (CBCT) are essential supports in finding root canal orifices. ${ }^{13}$

The morphological third molars depicted as erratic. ${ }^{14}$ nevertheless, restorative, orthodontics \& prosthetic concerns of these third molars require root canal treatment in turn to be retained as functional elements in the jaw. ${ }^{15,16,17}$

The third molar have to be considering as a strategic abutment is often causing loss of the mandibular 1st and 2nd molars. One more indication for endodontic treatment \& proper management is crucial for complete functioning of mandibular third molar in the jaw that has adequate space for eruption completely. ${ }^{18}$

The rationale of this study was to undertaken for revealing the root canal morphology of mandibular third molars to provide the better understanding to dentist to look forward when these teeth treating endodontically.

\section{METHODOLOGY}

This cross-sectional study was conducted at Department: Operative Dentistry, Faculty of Dentistry, Liaquat University of Medical \& Health Sciences, Jamshoro. Total 200 patients enrolled. All are mandibular 3rd molar (fully erupted in the jaw) Were endodontical treated using conventional stainless steel hand files by postgraduate trainee from February 2014 to March 2016.

\section{Inclusion criteria}

- Age group gender patients both male and female (31-40years)

- Complete erupted mandibular third molars along with History of irreversible pulpitis undergoing endodontic treatment.

\section{Exclusion criteria}

- Inadequate mouth opening,

- Grossly carious mandibular third molar,

- Improperly erupted 3rd molar either bacco or lingual version.

- Severely curved roots radiographically and

- Mentally retard handicapped patients.

Consent form along with demographic information was obtained. Standard procedures for root canal therapy containing history taking, preoperative radiograph, local anesthesia and isolation were maintained. Than access was made to cavity by opening the pulp chamber in order to give direct access to the apical foramina by removing the roof of the pulp chamber. Started Opening of pulp chamber was done with the round than inverted cone burs in a contra-angle high speed hand piece, was directed towards the axis of largest canal. Enough roof of the pulp chamber was removed to assist easy access for exploration of root canal. On this step, entire carious structure of tooth was removed to decrease the chances for re-infection.

Clinically examining the floor of pulp chamber for searching the canal orifices with the help of endodontic explorer and then placing files in all the canals located for confirming number of canals and taken radiograph for obtaining the working length after that canals were instrumented by step down method for widening the orifices of the canal using Gates Glidden drills and canals prepared by K-files. Obturation was done by the lateral condensation technique and then permanently restored the tooth with amalgam restoration. Post operatively radiograph was taken after completion of the obturation.

The collected data is entered into SPSS version 20.0 and evaluated by its statistical package. The qualitative variables like gender and patients having various numbers of canals $(1,2,3$ or 4$)$ are obtained by means of frequency and percentage and presented in charts.

\section{RESULTS}

Total 200 root canal treatment were done of lower 
third molar with either side, out of 200 patients 135 (67.5\%) were males and remaining 65 (32.5\%) were females, the age group commonly presented at Operative Dentistry department for lower third molar RCT were 31-40 years of age 93 $(46.5 \%)$, followed by $41-50$ years $40(20 \%), 20$ 30 years 35 (17.5\%), 51-60 years $32(16 \%)$. As shown in Figure-1 and 2.

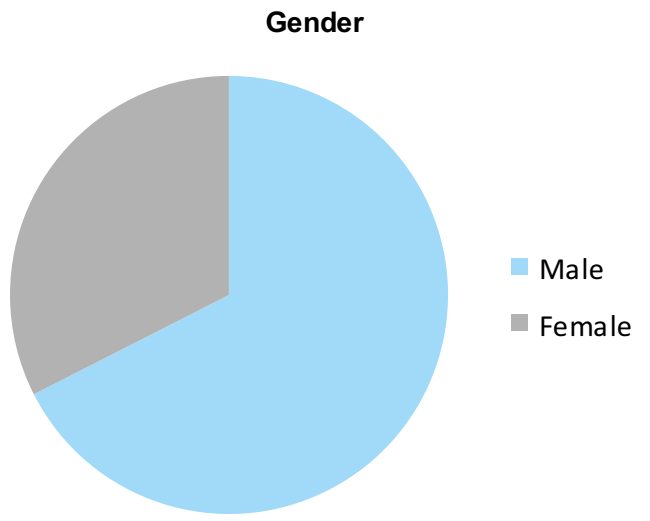

Figure-1. Showing the gender distribution

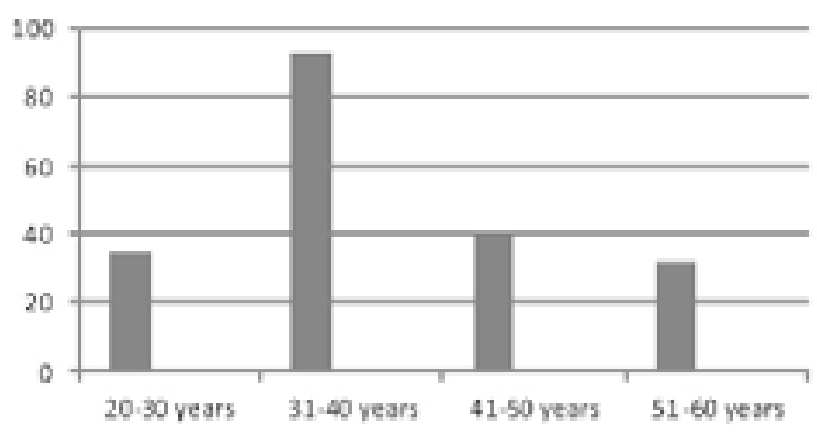

Figure-2. Showing the age group distribution

Figure-3 showing the level of post graduate residents were working at Operative Dentistry department; first year of training R1- 25 (12.5\%), second year of training R2- 35 (17.5\%), third year of training R3- $60(30 \%)$, and final year of training R4- 80 (40\%). As shown in Figure-3.

Out of the 200 conventionally RCT treated lower third molar teeth, we were found to have a practical error like under fill 25 (12.5\%), followed by overfill $20(10 \%)$, instrument separation $10(05 \%)$, ledge formation 10 (05\%) and remaining 135 (67.5\%) were normal RCT treated. As shown in Figure-4.
Figure-5 showing the number of canal configuration in lower third molar teeth, the one canal were present in $4(2.0 \%)$ teeth, followed by 2 canals in $33(16.5 \%)$ teeth, 3 canals in 160 $(80 \%)$ teeth and 4 canals in $3(1.5 \%)$ teeth.

\section{Status of Post graduate Students}

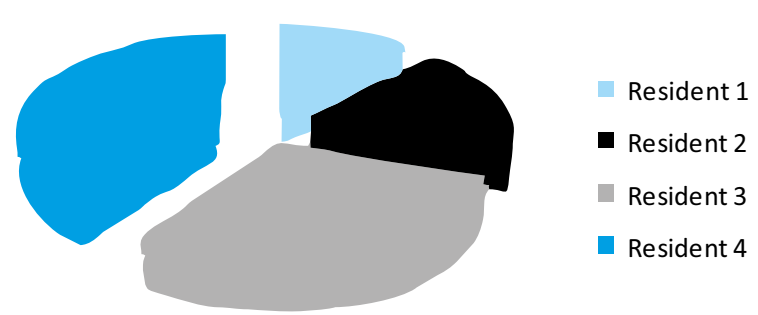

Figure-3. Showing the level of residence in training

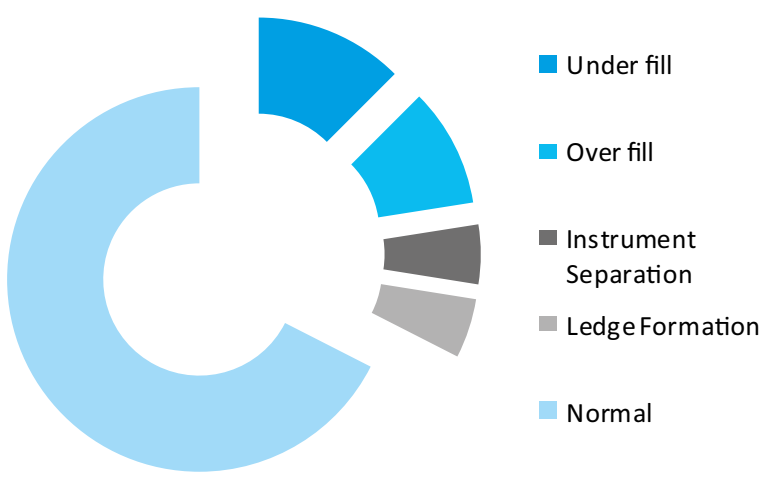

Figure-4. Showing the Conventional RCT errors

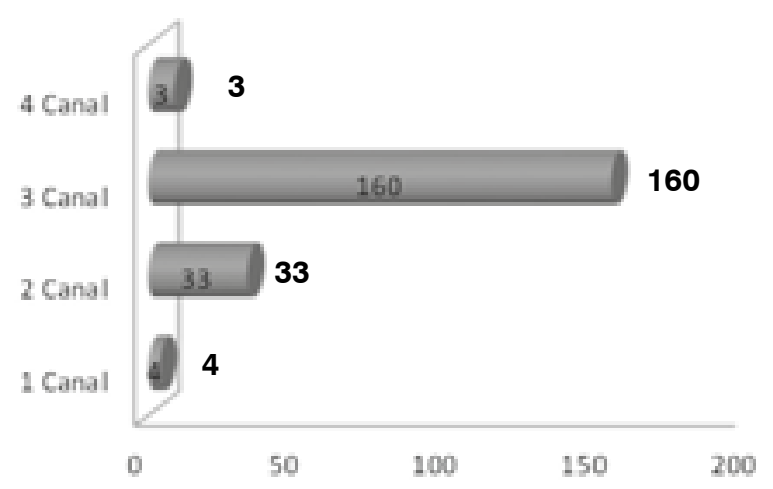

Figure-5. Canal configurations of lower third molar teeth

\section{DISCUSSION}

Successful endodontic therapy based on diagnosticmeasures, treatmentplanning, scientific 
skill and knowledge of typical morphology of the pulp canal system. Before undertake root canal treatment, it's very essential to visualized and wellknown for inner anatomical relationships of root canals. Two or more radiographs are mandatory for assessment, currently spiral computed tomography (SCT), being used for treatment \& designing for dental implants, it is better than staining and clearing technique in root canal structure \& morphologic studies. ${ }^{19-23}$ This study has undertaken for revealing the morphology of mandibular third molars to provide the better understanding to dentist to look forward when these teeth treating endodontically.

This study illustrate that out of 200 patients $67.5 \%$ were male and $32.5 \%$ were female. Majority of cases belong with age group 31-40years 93 (46.5\%), followed by $41-50$ years 40 (20\%), 20-30 years $35(17.5 \%), 51-60$ years 32 (16\%). maximum cases were done by 3rd year (R3) or final year (R4) of post graduate students during procedure, endodontic procedural errors were encountered in around 65 cases like underfill (25), overfill (20), instrument separation (10), ledge formation (10) and the rest of cases 135 were done successful endodontic treatment without any endodontic errors.

Canal configuration in this study were found one canal in four cases ,two canals in thirty three cases, and my study result did not directly support to the result of studies done by Kuzekanani $\mathrm{M}$ et al. ${ }^{18}$ in southeast of Iran, study by Sidow SJ. ${ }^{24}$ Three canals seen in the one hundred sixty cases. One of the study done by four canals had been found in three cases of root canal treatment so majority of patients having 3 canals. one of the study carried out by Guerisoli et al in contrast with study illustrated that one root canal had seen in $12.3 \%$ patients. ${ }^{24,25}$

We didn't detect any mandibular third molar having 5 or 6 root canals. And these results were similar with the study done by Kuzekanani $M$ et al. ${ }^{18}$ but a study conducted by Sinha DJ et al in the year 2014, their result clearly indicate five canals. ${ }^{26}$

\section{CONCLUSION}

Morphological features of mandibular 3rd molar are always unpredictable, and showed a discrepancy by way of different individual. In spite of being a common dental practice, least interference \& preserving each functional element of the jaw are most important in current dental procedures. Nevertheless, anatomical challenges, a proper awareness and complete clinical meticulousness is necessary to circumvent the incidence of severe problems whilst initiating endodontic treatment in the 3rd molar teeth. However restorative, prosthodontics, and orthodontic concerns required root canal treatment of third molars in array to be save the functional part in the dental arch. Mandibular third molars showed huge anatomic irregularity. Mandibular 3rd molar may have one or two roots. As it's a case series study Mandibular 3rd molar have up to four canals. Considerably gender has no any effect on number of root canals of mandibular third molars.

Copyright (C) 28 Feb, 2017.

\section{REFERENCES}

1. Oliver Valencia de Pablo, Roberto Estevez, Manuel P_eix S_anchez, Carlos Heilborn, et al. Anatomy and Canal Configuration of Permanent Mandibular First Molar. J Endod. 2010; 36:1919-31.

2. Schilder H. Filling root canals in three dimensions. Dent Clin North Am 1967; 723-44.

3. Schilder $\mathrm{H}$. Cleaning and shaping the root canal. Dent Clin North Am 1974; 18: 269-96.

4. Carrotte P. Endodontics: Morphology of the root canal system. Brit Dent J 2004; 197: 379-83.

5. Pattanshetti N, Gaidhane M, Alkandari AM. Root and canal morphology of the mesiobuccal and distal roots of permanent first molars in Kuwaiti population. Int Endod J 2008; 41:755-62.

6. Awawdeh LA, Al-Qudah AA. Root form and canal morphology of Mandibular premolars in a Jordanian population. Int Endod J 2008; 41: 240-48.

7. Gulabivala $\mathrm{K}$, Aung $\mathrm{TH}$, Alavi A. Root and canal morphology of Burmese mandibular molars. Int Endod J 2001; 34: 359-70.

8. Furri M. Differences in the con uence of mesial canals in man $\neg$ dibular molar teeth with three or four root 
canals. Int Endod J 2008; 41: 777-80.

9. Gulabivala K, Aung TH, Alavi A. Root and canal morphology of Burmese mandibular molars. Int Endod J 2001; 34: 359-70.

10. Plotino G. A mandibular third molar with three mesial roots: A case report. J Endod 2008; 34: 224-26.

11. Abella F, Teixido LM, Patel S, Sosa F, Sindreu FD, Roig M. Cone-beam Computed Tomography Analysis of the root canal morphology of maxillary first and second premolars in a Spanish population. J Endod 2015; 41: 1241-47.

12. Fabra-Campos $\mathrm{H}$. Three canals in the mesial root of mandibular first permanent molars: a clinical study. Int Endod J 1989; 22:39-43.

13. Vertucci FJ. Root canal morphology and its relationship to endodontic procedures. Endod Topics 2005; 10: 3-29.

14. Hargreaves KM, Cohen S. Pathways of the Pulp, 10th ed. Missouri: Mosby; 2011:215

15. Olson DJ, Roberts S. Unevenness of the apical construction in human maxillary central incisors. J Endod 2008; 34:157-9.

16. Baratto F, Zaitter $\mathrm{S}$. Analysis of the internal anatomy of maxillary first molars by using different methods. $J$ Endod 2009; 35:337-42.

17. Martos J, Lubian C. Morphologic analysis of the root apex in human teeth. $\mathrm{J}$ Endod 2010; 36:664-7.

18. Kuzekanani M, Haghani J, Nosrati H. Root and Canal Morphology of Mandibular Third Molars in an Iranian Population. JODDD, Vol. 6, No. 3, 2012.
19. Reuben J, Velmurugan $\mathrm{N}$. The evaluation of root canal mor-phology of the mandibular first molars in an Indian popula-tion using spiral computed tomography scan: an in vitro study. J Endod 2008; 34:212-15.

20. Neelakantan P, Subbarao C, Subbarao CV. Comparative evaluation of modified canal staining and clearing technique, cone-beam computed tomography, pe plain and contrast medium-enhanced digital radiography in studying root canal morphology. J Endod 2010; 36:1547-51.

21. Deepalakshmi M, Miglani R, Indira R, Ramachandran S. Spiral CT diagnosis and endodontic management of an ana-tomically variant palatal root with two canals in a maxillary first molar. Indian J Dent Res 2010; 21:443-5.

22. Meder C, Cowherd L, Williamson AE, Johnson WT. Apical morphology of the palatal roots of maxillary molars by using micro-computed tomography. J Endod 2011; 37:1162-5.

23. Yamada $M$, Ide $Y$, Matsunaga $S$, Kato $H$. Three dimensional analysis of mesiobuccal root canal of Japanese maxillary first molar using Micro-CT. Bull Tokyo Dent Collkyo Dent Coll 2011;52:77-84.

24. Sidow SJ, West LA, Liewehr FR, Loushine RJ. Root canal morphology of maxillary and mandibular third molars. J Endod 2000; 26: 675-78.

25. Guerisoli DMZ, Souza RA, Neto MDS, Silva RG, Pecora JD. External and internal anatomy of third molars. Braz Dent J 1998; 9: 91-94.

26. Sinha DJ, Sinha AA. An endodontic management of mandibular third molar with five root canals. Saudi Endod J 2014; 4:36-9.

\section{PREVIOUS RELATED STUDY}

Faisal, Sana Shah, Naresh Kumar, Syed Abrar Ali, Nadia Inayat. Root canal treatment (Original) Professional Med J Sep-Oct 2012;19(5): 739-741.

\section{AUTHORSHIP AND CONTRIBUTION DECLARATION}

\begin{tabular}{c|l|l|l|} 
Sr. \# & \multicolumn{1}{|c|}{ Author-s Full Name } & \multicolumn{1}{|c|}{$\begin{array}{c}\text { Contribution to the paper } \\
\text { Author=s Signature }\end{array}$} \\
\hline 1 & Dr. Naveen Khawaja & $\begin{array}{l}\text { Data collection \& } \\
\text { Methodology } \\
\text { Introduction \& Discussion }\end{array}$ \\
\hline 3 & Dr. Suneel Kumar Punjabi & Dr. Munir Ahmed Banglani & Abstract \& Proof reading \\
\hline
\end{tabular}

